\title{
The Aggregation Problem in the Employment Theory: The Representative Individual Model or Individual Employees Model?
}

\author{
Masayuki Otaki \\ Institute of Social Science, University of Tokyo, Tokyo, Japan \\ Email: ohtaki@iss.u-tokyo.ac.jp
}

Received June 7, 2012; revised July 8, 2012; accepted August 1, 2012

\begin{abstract}
Employment theory does lacks a consensus concerning whether employment variation should be expressed as a change in the hours worked as a representative individual or as a change in the population of employed individuals. By appling the OLG model developed by Lucas [1] and Otaki ([2-4]), the present article describes a serious theoretical consequence of distinction. The crucial factor that different employment theories are the intertemporal substitution effect and the indivisibility of labor force. Monetary expansion increases the rate of return for money if it is credible in the sense of Otaki [5]. This enhances the hours worked in the representative individual model, and thus, aggregate supply causes demand. Conversely, in the indivisible employees model, such an intertemporal substitution effect does not exist. The monetary expansion directly improves the purchasing power of money and thereby increases the aggregate demand for goods by the older generation. Thus, demand derives supply.
\end{abstract}

Keywords: Representative Individual; Indivisible Labor Supply; Intertemporal Substitution; Credibility of Money; Fiscal Multiplier

\section{Introduction}

Independent of whether researchers adopt neoclassical or new Keynesian economic models, recent employment theories have rested on the assumption of a representative individual. However, it is important to note that the hours worked by a representative individual differs crucially from indivisible employees who each work equal amount of time. In this paper, we show that such a distinction has serious theoretical consequences.

The crucial factor is the existence of the intertemporal substitution effect. In the representative model, an expansion of money raises the rate of return as long as money is credible and stimulates the labor supply. Hence, apart from the spurious difference, both neoclassical and new Keynesian models seek the cause of employment variation for the supply-side incentive.

In contrast, there is no such substitution effect in the indivisible employees model ${ }^{1}$. A monetary expansion

\footnotetext{
${ }^{1}$ Although we can principally separate the adjustment of hours worked from that of employment level (see Fukao and Otaki [7]), doing so requires far more complex dynamics, which are not essential to our discussion. Furthermore, if there is no fixed sunk cost for being employed, it will be clarified that every firm uniformly offers minimal hours worked because the increasing marginal disutility of labor requires higher wages for compensation.
}

directly increases the purchasing power of money as Otaki [4] shows, even if the money-supply rule obeys that by Lucas [1] as long as money is credible. It also implies that the monetary expansion increasess the aggregate demand, which in turn increases the real GDP. That is, the demand causes the corresponding supply, as Keynes [6] observed.

The rest of the paper is organized as follows. Section 2 constructs alternative models concerning the employment theory. Section 3 contains brief concluding remarks.

\section{The Model}

\subsection{The Structure of the Model}

We consider a standard two-period deterministic OLG model in a production economy. In every period, a unit of individual is born. They can work only when they are young. A unit working hour produces unit goods.

The money supply obeys Lucas's [1] rule. That is,

$$
m_{t}=m_{t-1} x,
$$

where $m_{t-1}$ is the nominal money stock per capita that is carried over from the previous period. $x$ is the gross increase rate of money. In this sense, new money is supplied as its own nominal interest rate. 
We make the following alternative assumptions concerning the labor supply: 1) In the representative individual model, the representative individual can chooses his working hours and there is no unemployment problem; 2) In the indivisible employees model, each individual faces the discrete choice of whether to work.

\subsection{The Representative Individual Model}

\subsubsection{The Definition of Equilibrium}

For simplicity, we assume that the representative individual possesses the following utility function $U_{R}$ :

$$
U_{R} \equiv u\left(c_{1 t}, c_{2 t+1}\right)-v\left(h_{t}\right),
$$

where $u$ is a well-behaved linear homogenous function. $c_{1 t}, c_{2 t+1}$ denote the consumption level sof generation $t$ during the young and old stages of life, respectively. $h$ is the hours worked.

$v(h)$ has the following properties.

$$
v(h) \equiv\left\{\begin{array}{l}
0, h=0, \\
\varepsilon, 0<h \leq \underline{h}, \\
\phi(h), \underline{h} \leq h, 0 \leq \phi, 0<\phi^{\prime \prime}
\end{array}\right.
$$

The shape of $v(h)$ is illustrated in Figure 1.

The assumption that some lower limit $\varepsilon$ exists for the disutility of labor is equivalent to the assumption that individuals do not incur any additional disutility by increasing in hours worked to some extent. As the classical economists presume. Its economic meaning of this assumption is that there is an urgent need to produce goods that correspond to the subsistent level, as shown below.

The lifetime budget constraint is

$$
p_{t} c_{1 t}+m_{t-1} \leq p_{t} h_{t}, p_{t+1} c_{2 t} \leq m_{t-1} x \Leftrightarrow c_{1 t}+\frac{p_{t+1}}{p_{t} x} c_{2 t+1} \leq h_{t}
$$

Since the lifetime utility function concerning the consumption stream is concave and homothetic, we obtain the following correspond indirect utility function $\Psi$ :

$$
\Psi=\frac{p_{t} h_{t}}{\psi\left(p_{t}, \frac{p_{t+1}}{x}\right)}, \psi_{1}>0, \psi_{2}>0 .
$$

Moreover, we can ascertain that

$$
\left.\Psi^{\prime}\right|_{h_{t}=\underline{h}}-\left.\phi^{\prime}\right|_{h_{t}=\underline{h}}>0
$$

holds. This expression implies that, as long as $\varepsilon$ is sufficiently small, the equilibrium hours worked always

\footnotetext{
${ }^{2}$ The concept of the credibility of money is a device used to select a unique rational expectation equilibrium (REE) from among multiple REEs that are generic to the OLG model of the monetary economy. Credibility economically means that people rationally believe its intrinsic value is kept intact even if the velocity of monetary acceleration is changed.
}

exceeds the subsistent lower limit $\underline{h}$, and that the problem of the indivisibility of hours worked never appears in the decision problem.

The optimality conditions are

$$
\begin{gathered}
\frac{1}{\psi\left(1, \frac{p_{t+1}^{*}}{x p_{t}^{*}}\right)}-\phi^{\prime}\left(h^{*}\right)=0, \\
c_{1}^{*}=c^{*}\left(\frac{p_{t+1}^{*}}{x p_{t}^{*}}\right) h_{t}^{*}, \\
c_{2}^{*}=\frac{x p_{t}^{*}}{p_{t+1}^{*}}\left[1-c^{*}\left(\frac{p_{t+1}^{*}}{x p_{t}^{*}}\right)\right] h_{t}^{*},
\end{gathered}
$$

We assume, according to Lucas [1], that leisure and the current consumption are not inferior goods. Equation (6) directly implies that

$$
\frac{\left(c^{*}\right)^{\prime}}{c^{*}}+\frac{\left(h^{*}\right)^{\prime}}{h^{*}}<0 .
$$

In addition to the three optimality conditions, there is one independent market equilibrium condition. Here, we consider the condition for the money market equilibrium: that is,

$$
m_{t-1} x=p_{t+1}^{*} c_{2 t+1}^{*} \text {. }
$$

Furthermore, we assume the credibility of money in the sense of Otaki $[5]^{2}$ :

$$
\frac{\mathrm{d} p_{t+1}^{*}}{\mathrm{~d} x}=0 .
$$

There are five endogenous variables $\left(c_{1 t}^{*}, c_{2 t+1}^{*}, h_{t}^{*}, p_{t}^{*}, p_{t+1}^{*}\right)$, and five independent Equations (5)-(9). Hence, the model is closed, and the solution consists of a temporary rational expectation equilibrium.

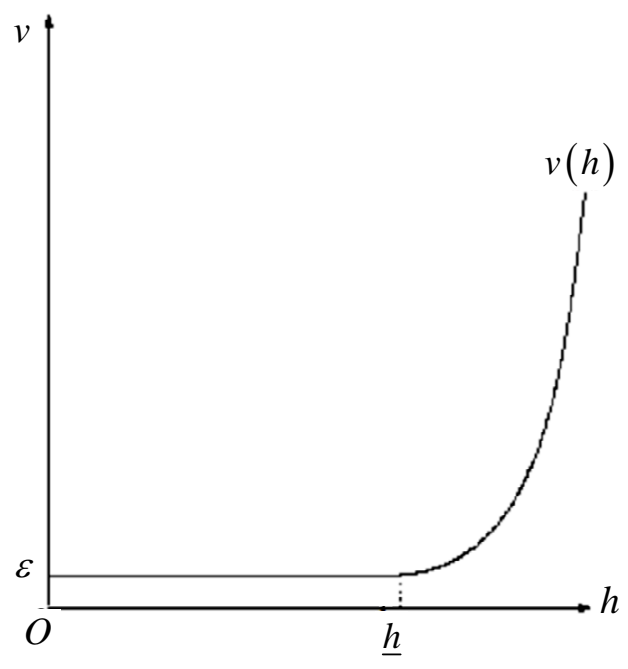

Figure 1. Disutility function of labor. 


\subsubsection{Comparative Statics}

From Equations (8) and (9), it is clear that $c_{2}^{*}$ increases with the nominal interest rate of money $x$. Equations (5) and (6) imply that $c_{2}^{*}$ is a monotonically decreasing function of the effective inflation rate (the inverse of the real interest rate) $\frac{p_{t+1}^{*}}{p_{t}^{*} x}$. Thus, $\frac{p_{t+1}^{*}}{p_{t}^{*} x}$ decreases as $x$ increases. It is also apparent from Equation (5) that equilibrium working hours $h_{t}^{*}$ increases with $x^{3}$.

To summarize, as long as money is credible, an easy monetary policy increases the real interest, and hence, the representative individual works more to enjoy more future consumption. Accordingly, a monetary expansion advances intertemporal substitution from current consumption and leisure into future consumption by raising the real rate of interest. As such, the expansionary effect of monetary policy is entirely based on the labor supply incentive, not on the expansion of the aggregate demand. In this sense, the representative individual model is inevitably classified as a neoclassical macroeconomic model.

\subsubsection{The Time-Independence of the Model}

Assume that the representative individual rationally expects that the real effective inflation rate $\frac{p_{t+1}^{*}}{p_{t}^{*} x}$ is kept intact after period $t+1$, since no economic environment is changed after period $t^{4}$. This assumption implies that

$$
\frac{\mathrm{d} x}{x}=\frac{\mathrm{d} p_{t+j+1}^{*}}{p_{t+j+1}^{*}}-\frac{\mathrm{d} p_{t+j}^{*}}{p_{t+j}^{*}}, \forall j \geq 0 .
$$

In addition

$$
m_{t+j-1} X=p_{t+j+1}^{*} c_{2 t+j+1}^{*} \text { and } m_{t+j-1}=p_{t+j}^{*} c_{2 t+j}^{*}
$$

holds from the money market equilibrium condition (8). Thus, we obtain

$$
\frac{\mathrm{d} c_{t+j+1}^{*}}{c_{t+j+1}^{*}}-\frac{\mathrm{d} c_{t+j}^{*}}{c_{t+j}^{*}}=\frac{\mathrm{d} x}{x}-\left[\frac{\mathrm{d} p_{t+j+1}^{*}}{p_{t+j+1}^{*}}-\frac{\mathrm{d} p_{t+j}^{*}}{p_{t+j}^{*}}\right]=0, \forall j \geq 0 .
$$

That is, future consumption $C_{2}^{*}$ becomes time-independent. Hence, from Equations (5)-(7), $\left(c_{1}^{*}, h^{*}\right)$ are also time-independent. Consequently, the rational expectation equilibrium characterized by the initial condition $x$ and the expectation formulation (9) and (10) are stationary.

\subsection{The Indivisible Employees Model}

Here, we assume that labor supply is indivisible, and that each individual has the identical utility function $U_{I}$ :

${ }^{3}$ Otaki [4] has already proved such a property in the different type of utility function, although that work does not consider the serious aggregation problem addressed in this paper.

${ }^{4}$ Note that this assumption contains the credibility of money as the case for $j=0$.

$$
U_{I} \equiv u\left(c_{1 t}, c_{2 t+1}\right)-\delta_{t} v\left(h_{t}\right),
$$

where $u$ is the same consumption utility function as in Equation (1). $\delta_{t}$ denotes a definition function that takes the value unity when the individual works unit time and that is zero when the individual does not work.

According to Equation (1), the nominal minimal revenue that individuals decide to work $(N R)$ is represented as

$$
N R=p_{t}^{*} \psi\left(1, \frac{p_{t+1}^{*}}{x p_{t}^{*}}\right) \varepsilon
$$

We must note that firms strictly prefer increasing employment to the upward adjustment in working hours per capita in any interior equilibrium in which unemployment exists and all individuals are indifferent to the decision of whether to work.

The reason is as follows. Even if a unit employment increases, as long as the working hours per capita are fixed, there is no appreciation of nominal wages. However, the concavity of $\phi$ requires nominal wages higher than $N R$ to induce working hours that produce the same amount of output as in the case of employment adjustment. Thus, as long as unemployment exists, working hours per capita is fixed to the minimal level $\underline{h}$.

Accordingly, we obtain the following difference equation concerning the evolution of price sequence ${ }^{5}$ :

$$
p_{t+j}^{*} \underline{h}=\psi\left(p_{t+j}^{*}, \frac{p_{t+j+1}^{*}}{x}\right) \varepsilon \Rightarrow 1=\psi\left(1, \frac{p_{t+j+1}^{*}}{p_{t+j}^{*} x}\right) \frac{\varepsilon}{\underline{h}}, 0 \leq j .
$$

Hence, the equilibrium real interest rate

$$
r^{*} \equiv \frac{p_{t+j}^{*} x}{p_{t+j+1}^{*}}
$$

is independent of $x$ and takes a constant value.

The equilibrium condition for the money market is

$$
s\left(r^{*}\right) y_{t+j}^{*}=\frac{m_{t+j-1} X}{p_{t+j+1}^{*}} \equiv \tilde{m}_{t+j}^{*}, 0 \leq j,
$$

where $s(\cdot)$ is the marginal propensity to save.

Assuming the credibility of money (i.e., $\frac{\mathrm{d} p_{t+1}^{*}}{\mathrm{~d} x}=0$ ), an increase in the monetary growth rate $x$ increases the current value of money and empowers the purchasing power of old individuals as long as money is a credible asset. As such, the monetary expansion stimulates the economy through the multiplier effect developed by Otaki [2].

It is not difficult to show the time-independence of the

${ }^{5}$ See Otaki [5], for the case in which the equilibrium is located at boundaries. 
equilibrium. Assume that people rationally believe that the price level $p_{t+j+1}^{*}$ grows proportionately with the monetary expansion rate $x$, that is,

$$
\frac{\mathrm{d} p_{t+j+1}^{*}}{p_{t+j+1}^{*}}=\frac{\mathrm{d} x}{x}, 0 \leq j .
$$

Then, the equilibrium real cash balance $\tilde{m}^{*}$ becomes time-independent, so does the real equilibrium GDP $y^{* 6}$.

In addition, as we previously mentioned, the individual employees model is similar to a Keynes' [6] type model in the sense that a monetary expansion stimulates the aggregate demand unlike the representative individual model that crucially relies on the supply-side intetemporal substitution incentive.

\section{Concluding Remarks}

This article analyzed how the aggregation problem affects the theory of employment. We obtained the following results.

First, because of the intertemporal substitution between goods and leisure, a change in working hours in the representative individual model is supply-side oriented even if money is credible and non-neutral. Furthermore, since it does not contain the concept of indivisibility of labor, this model cannot explain why unemployment occurs although it can spuriously trace the total output movement. An accelaration in monetary growth increases the real interest of money, and thus, intertermporal substitution occurs from leisure and current consumption to future consumption.

Second, the indivisible individual employees model possesses the demand-driven property deepened by Keynes [6]. Although the real rate of interest of money is en- dogenously fixed, whenever money is credible, money comes to be highly valuated and raises the purchasing power of the old generation via the acceleration of monetary growth. As such, the effective demand expands, and the real GDP increases via the multiplier effect.

In summation, Keynes' [6] economics can be characterized by the following two factors. The first is the credibility on a fiat money of which intrinsic value is basically indetereninate. Second is the specificity of labor as a commodity, namely, the indivisibility of labor.

\section{REFERENCES}

[1] R. E. Lucas Jr., "Expectations and the Neutrality of Money," Journal of Economic Theory, Vol. 4, No. 2, 1972, pp. 103-124. doi:10.1016/0022-0531(72)90142-1

[2] M. Otaki, "The Dyanamically Extended Keynesian Cross and the Welfare-Improving Fiscal Policy," Economics Letters, Vol. 96, No. 1, 2007, pp. 23-29. doi:10.1016/j.econlet.2006.12.005

[3] M. Otaki, "A Welfare Economic Foundation for the Full Employment Policy,” Economics Letters, Vol. 102, No. 1, 2009, pp. 1-3. doi:10.1016/j.econlet.2008.08.003

[4] M. Otaki, "The Role of Money: Credible Asset or Numeraire?” Theoretical Economics Letters, Vol. 2, No. 2, 2012, pp. 180-182. doi:10.4236/tel.2012.22031

[5] M. Otaki, "A Pure Theory of Aggregate Price Determination,” Theoretical Economics Letters, Vol. 1, No. 3, 2011, pp. 122-128. doi:10.4236/tel.2011.13026

[6] J. M. Keynes, “The General Theory of Employment, Interest and Money,” Macmillan, London, 1936.

[7] K. Fukao and M. Otaki, "Accumulation of Human Capital and the Business Cycle,” Journal of Political Economy Vol. 101, No. 1, 1993, pp. 72-99. doi:10.1086/261866

\footnotetext{
${ }^{6}$ Note that Equation (16) is also compatible with the assumption of credibility of money, because the equilibrium price during $t+1$ increases by $\frac{\mathrm{d} x}{x}$ relative to the newly lowered equilibrium current price $p_{t}^{*}$.
} 